\title{
Symmetry-breaking on-off intermittency under modulation: Robustness of supersensitivity, resonance and information gain
}

\author{
Bambi $\mathrm{Hu}^{1,2}$ and Changsong Zhou ${ }^{1}$ \\ ${ }^{1}$ Department of Physics and Center for Nonlinear Studies, Hong Kong Baptist University, Hong Kong, China \\ ${ }^{2}$ Department of Physics, University of Houston, Houston, Texas 77204
}

\begin{abstract}
Nonlinear dynamical systems possessing an invariant subspace in the phase space and chaotic or stochastic motion within the subspace often display on-off intermittency close to the threshold of stability of the subspace. In a class of symmetric systems, the intermittency is symmetry-breaking [Ying-Cheng Lai, Phys. Rev. E 53. R4267 (1996)]. We report interesting and practically important universal behavior of robustness of supersensitivity, resonance and information gain in this class of systems when subjected to a weak modulation. While intermittent loss of synchronization may be harmful to application of high-quality synchronization of coupled chaotic systems, the features reported here may lead to interesting application of on-off intermittency.
\end{abstract}

PACS number(s): 05.40.-a, 05.45.-a

\section{INTRODUCTION}

Nonlinear dynamical systems possessing an invariant subspace are of great interest, particularly when the system motion within the subspace can be chaotic or stochastic. Examples include chaotic systems with symmetry [1,2] or coupled chaotic systems [3]. Bubbling [4, [5] and on-off intermittency [6] are typical behaviors in system close to a threshold of transverse stability of the subspace due to the fluctuative nature of the local transverse Lyapunov exponent in different part of the subspace. In bubbling, the subspace is stable on average, but local instability can result in large bursts away from the subspace when it is perturbed. The sensitivity of this weak stability to parameter mismatch and noise has been studied by Pikovsky and Grassberger [7]. Intermittent loss of synchronization [5] in experiments with inevitable noise and parameter mismatch is undesirable in application of highquality synchronization, such as to communication [8]. In on-off intermittency, the subspace is slightly unstable on average, but local transverse attraction may keep the dynamics very close to the subspace for a long period of time. Great attention has been paid to the duration of this laminar period which exhibits universal power law distribution in a broad class of systems 6. Noise in the system prevents its state from approaching the subspace close beyond the noise level, thus has important effects on the laminar period distribution [9] or the escape time [10]. In noisy environment, bubbling and on-off intermittency are essentially the same phenomenon.

Sensitivity in nonlinear systems can be very useful for applications such as controlling global dynamics of the system by local tiny perturbations [11]. It is interesting to ask whether the sensitivity of on-off intermittency may lead to any potential application of the phenomenon. An observation is that in a class of symmetric systems, on-off intermittency can be symmetry-breaking [i], namely, the bursting behavior does not possess the system symmetry when the system has two symmetric but distinct attractors. This Letter reports that a combination of the sensitivity of on-off intermittency and the symmetry-breaking of the bursting can result in remarkable features in the systems subjected to a weak modulation signal in the noisy environment.

\section{THE MODEL}

Let $x(t)$ represent the distance of the dynamics from the invariant subspace, and $x(t)>0$ and $x(t)<0$ denote the dynamics in the two symmetric components respectively. In the symmetry-breaking systems, transitions between $x>0$ and $x<0$ can only occur when $x(t)$ comes to the level of the weak noisy signal. For system displaying appreciable laminar state, main features of the dynamics can be described by the general linear equation close to the subspace:

$$
\dot{x}(t)=\left[\lambda+\sigma_{1} \xi(t)\right] x(t)+\sigma_{2} e(t)+p s(t) .
$$

Here $\lambda$ is the transverse Lyapunov exponent of the subspace, and $\sigma_{1} \xi(t)$ with $\langle\xi(t)\rangle=0$ is the fluctuation of the local Lyapunov exponent due to the chaotic or stochastic motion within the subspace. In general, chaotic system has quickly decaying correlation, and in a large enough time scale $t, \xi(t)$ has an asymptotic Gaussian distribution. $e(t)$ is the additive white noise with level $\sigma_{2} \ll \sigma_{1}$ and $s(t)$ is a weak modulation signal. The exact form of the signal is unimportant for the phenomena reported below, provided it varies on a time scale slower than the characteristic times of the systems. Here we consider $s(t)$ a random binary stream $( \pm 1$ with probability 0.5$)$ with a bit duration T. $p \ll \sigma_{1}$ is the amplitude of the signal, and $R=p / \sigma_{2}$ provides a natural measure of the signal-to-noise ratio (SNR).

In Ref. [10], Cenys and Lustfeld studied the statistical properties of escape time of on-off intermittency sub- 
jected to noise by means of Fokker-Planck equation. It has been shown that on-off intermittency is very sensitive to noise [9,10]. We employ the same approach of Fokker-Planck equation, and focus on the property of amplification of the weak external signal $s(t)$ in the system. Our results will demonstrate that the system is also very sensitive to the weak signal, and the amplification of the weak signal is robust to the additive noise. This sensitivity exhibits resonant behavior as the system parameters change.

The Fokker-Planck equation for Eq. (11) is

$$
\begin{aligned}
\frac{\partial W}{\partial t} & =-\frac{\partial}{\partial x}\left\{\left[\left(\lambda+\frac{\sigma_{1}^{2}}{2}\right) x+p s(t)\right] W\right\} \\
& +\frac{1}{2} \frac{\partial^{2}}{\partial x^{2}}\left[\left(\sigma_{1}^{2} x^{2}+\sigma_{2}^{2}\right) W\right] .
\end{aligned}
$$

In general, it is quite difficult to solve Eq. (2) exactly. Let $T_{0}$ be the relaxation time of the system after $s(t)$ switching from $+1(-1)$ to $-1(+1)$. If $T \gg T_{0}$, the probability distribution $W(t)$ can establish an approximate static state during each bit of the input signal. Under the adiabatic approximation $T \gg T_{0}, \partial W / \partial t \approx 0$, and the static solution can be obtained analytically as

$$
W(x)=C\left(x^{2}+\frac{\sigma_{2}^{2}}{\sigma_{1}^{2}}\right)^{(\alpha-1) / 2} \exp \left[\frac{2 p s(t)}{\sigma_{1} \sigma_{2}} \arctan \frac{\sigma_{1} x}{\sigma_{2}}\right]
$$

where $\alpha=2 \lambda / \sigma_{1}^{2}$. For $\left|\sigma_{1} x / A\right| \gg 1\left(A=\max \left(p, \sigma_{2}\right)\right)$,

$$
W(x) \approx C|x|^{\alpha-1} \exp \left[\frac{\pi p s(t)}{\sigma_{1} \sigma_{2}} \operatorname{sgn} x\right] .
$$

Now we see that the behavior of the system can be divided into two regimes. One is $|x| \gg\left|\sigma_{2} e(t)+p s(t)\right|$, where the dynamics is governed approximately by $\dot{x}(t)=$ $\left[\lambda+\sigma_{1} \xi(t)\right] x(t)$. Let $z=\ln |x|$, then $\dot{z}(t)=\lambda+\sigma_{1} \xi(t)$ which describes a Brownian motion with a constant drift $\lambda$ and diffusion constant $\sigma_{1}^{2} / 2$. The nonlinearity of the system can be modeled by an effective reflecting boundaries of the Brownian motion at $\pm x_{b}$, which is of the order of $\sigma_{1}$. The probability density has a power form $W(x)=|x|^{\alpha-1}$, but is asymmetric for $x>0$ and $x<0$ in the presence of $s(t)$. The system can rarely perform transition between $x>0$ and $x<0$ in this regime due to the symmetry-breaking property, until it comes to the other regime, where the noisy input $\sigma_{2} e(t)+p s(t)$ dominates the dynamics and the system performs transition between $x>0$ and $x<0$ frequently. The behavior of the system is determined by the competition between the diffusion and the drift of the Brownian motion. If the drift time $t_{b}=\ln \left(\sigma_{1} / A\right) /|\lambda|$ is much smaller than the diffusion time $t_{d}=2 \ln ^{2}\left(\sigma_{1} / A\right) / \sigma_{1}^{2}$, the drift is dominant over the diffusion, and the system will either come to a metastable state induced by the noisy input for $\lambda<0$, or approach some state away from the invariant subspace for $\lambda>0$.
In both cases, the weak noisy input has no significant effects on the system behavior, i.e. the system is insensitive to the modulation. On the other hand, the diffusion is dominant for $t_{b} \gg t_{d}$, and the system can have access to both the level of the weak input and the boundary of the nonlinearity, exhibiting typical on-off intermittency and sensitivity to the weak modulation. Variation of the parameter $\lambda$ or $\sigma_{1}$ affects the competition between the drift and the diffusion, and the system is expected to display optimal response to the weak modulation with resonant characterization.

\section{ROBUSTNESS OF SUPERSENSITIVITY TO THE WEAK SIGNAL}

Let us consider the diffusion dominant region close to the critical point of the stability of the subspace, e.g. $|\lambda| \ll 1,\left|\alpha \ln \left(x_{b} / A\right)\right| \ll 1, \sigma_{1} \sim 1, p \sim 10^{-m}(m \gg 1)$. Employing the approximation in Eq. (ब1), and the effective reflecting boundaries at $\pm x_{b}$, the ensemble average $\langle x(t)\rangle$ is estimated as

$$
\langle x(t)\rangle \approx s(t) \frac{x_{b}}{\ln \left(x_{b} / A\right)} \tanh \frac{\pi R}{\sigma_{1}},
$$

which in the noise-free limit $\sigma_{2} \rightarrow 0$, assumes the form

$$
\langle x(t)\rangle \approx s(t) \frac{x_{b}}{\ln \left(x_{b}\right)-\ln p} .
$$

A logarithmical dependence of $\langle x(t)\rangle$ on the input level $p$ means an amplification of the weak signal $p s(t)$ with an factor $\langle x\rangle / p \sim 10^{m} / m(m \gg 1)$, i.e. the system exhibits supersensitivity to extremely weak modulation close to the critical point. This sensitivity was also reported in an overdamped Kramers oscillator with multiplicative noise free from additive noise $\left(\sigma_{2}=0\right)$, which is a specific example in this class of systems 12. In the absence of $s(t)$, the system produces symmetric bursting pattern with $\langle x(t)\rangle=0$; while the bursting pattern is reorganized to manifest the weak signal after it is fed into the system (see Fig. 1). The most interesting and practically important property is that the weak signal is manifested even buried in a relatively high level of noise, namely, the robustness of the supersensitivity. This behavior originates from the symmetry-breaking of the on-off intermittency in the system.

To demonstrate the above analysis, we employ the following system in simulations [1]

$$
\begin{aligned}
& \ddot{y}=-\gamma \dot{y}+4 y\left(1-y^{2}\right)+f_{0} \sin \omega t, \\
& \dot{x}=(a+b y) \sin (x)-x+\sigma_{2} e(t)+p s(t),
\end{aligned}
$$

where $y$ constitutes the forced Duffing chaotic oscillator. With $\gamma=0.05, f_{0}=2.3$ and $\omega=3.5$, the Duffing system is chaotic and $\sigma_{1} \approx 0.964 b$. The nonlinearity of the variable $x$ is related to an experimental model of superconducting quantum interference device (SQUID) [13]. 
However, we should stress that the specific form of the nonlinearity is of no importance for the phenomena. The transverse Lyapunov exponent of the invariant subspace $x=0$ is $\lambda=a-1$ due to the symmetry of the Duffing chaotic attractor. Fig. 1 shows typical behavior of the system and good agreement between the analytical and the simulation results for $\langle x(t)\rangle$ as a function of $R$. The agreement demonstrates that the general stochastic model in Eq. (1) gives good account for this type of system even though the motion in the subspace is deterministic chaos.

\section{RESONANT BEHAVIOR}

It is difficult to perform generally a quantitative analysis of the system response to the weak signal based on the linear dynamics in Eq. (11) as parameters $\lambda$ or $\sigma_{1}$ changes, because the effective boundary $x_{b}$ changes with the nonlinearity and the linear dynamical model with an effective reflecting boundary is often not sufficient to capture the dynamical property if $\alpha$ is appreciately positive. Moreover, as the parameters change, the relaxation time $T_{0}$ may become comparable to the bit duration $T$, and the transient behavior plays an important role in the system response and an adiabatic approximation is not valid any more. To demonstrate the resonant properties, we rely on simulations with the system in Eq. (7), while the Brownian motion model can provide a qualitative understanding of the properties, thus showing that the properties are generic and universal for a general class of the systems.

For a system with on-off intermittent output $x(t)$, the ensemble average $\langle x(t)\rangle$ and the correlation between $s(t)$ and $x(t)$ is relatively small even for the noise-free case $\sigma_{2}=0$, due to the power law fluctuation of $x(t)$. To better characterize the response of the system to the modulation, we transfer the output series $x(t)$ into a binary stream $X(t)$ by a threshold crossing process: suppose $x(t)$ becomes larger than a prescribed threshold $x_{t h}$ at some moment, after that $X(t)$ will keep at $X(t)=1$ until $x(t)$ crosses $-x_{t h}$ at another moment; $X(t)$ will not switch back from $X(t)=-1$ to +1 until $x(t)$ crosses $x_{t h}$ again, and so on. This binary presentation captures the most important feature of the transition of the bursting pattern between the two symmetric attractors. $X(t)$ has a strong correlation with $s(t)$ for weak noise case $\sigma_{2}<p$ if the system is close to the critical point. The exact value of $x_{t h}$ is not crucial for the properties described below. In the following, we fix $p=10^{-7}, T=2000$ and $x_{t h}=1$, and take the cross-correlation function $C$ between $s(t)$ and $X(t)$ estimated using $10^{4}$ bits of a random stream of $s(t)$ to demonstrate the resonant behavior in the system.

(1) With the change of $\lambda$. For $\lambda$ rather below the critical point $\lambda=0$, the system has a metastable state close to the level of the noisy input, and the diffusion is not strong enough to produce large bursts frequently enough,
$C$ will be small. On the opposite, if $\lambda$ is rather above the critical point, the drift is also dominant so that the system can seldom access to the level of the weak modulation, and becomes insensitive to the switching of $s(t)$ between \pm 1 , resulting in a small $C$ again. Close to the critical point, the system can access to the level of the weak signal and produce large bursts frequently due to strong enough diffusion. The switching of the weak signal is "sensed" and manifested by asymmetrical bursting to $x>0$ and $x<0$, giving an optimal value of $C$. This precess is illustrated by $C$ as a function of $\lambda$ in Fig. 2 for various $R$ values.

(2) With the change of $\sigma_{1}$. For a small $\sigma_{1}$ where the drift is dominant over diffusion, the system is not sensitive to the weak noisy input and $C$ assumes a small value. With the increase of $\sigma_{1}$, the diffusion becomes stronger, resulting in a smaller relaxation time $T_{0}$ and more frequent large bursts, and the system becomes more sensitive to the weak input. In the noise-free case $\sigma_{2}=0$, the increased sensitivity enables $X(t)$ to keep closer in phase to the weak signal $s(t)$ and $C$ approaches closer to 1.0 if the system maintains to work in the symmetrybreaking regime, and in general a resonant behavior is not expected. The picture becomes quite different if $\sigma_{2} \neq 0$. With smaller $T_{0}$ and increased sensitivity, the system can keep up with and manifest more and more noise-induced transitions in shorter time-scales, and the transition rate of $X(t)$ between \pm 1 may become much higher than that of $s(t)$, leading to a decreasing $C$. An optimal response is achieved when the diffusion is strong enough to become sensitive to the weak input but not too strong to manifest a lot of noise-induced transitions in short time-scales. Typical example of the system response as a function of $\sigma_{1}$ is shown in Fig. 3.

The above resonant behavior is similar to the conventional stochastic resonance where a dynamical system displays increased sensitivity to a subthreshold signal with an optimal level of additive noise, see Ref. 14. for an extensive review. Resonance occurs when a noisecontrolled time-scale in the system matches that of the signal. In our system, the underlying mechanism of the resonant behavior is quite different. The sensitivity to an extremely weak signal is induced by the multiplicative chaotic or stochastic motion in the subspace. To achieve this sensitivity, it is required that the system is in the on-off intermittency regime so that it can become susceptible to the weak signal by coming close enough to the subspace, and manifest and amplify it by quick enough large bursts away from the subspace with symmetrybreaking. As system parameters $\lambda$ and $\sigma_{1}$ change, a competition between these two factors leads to the resonant behavior. More interestingly, resonant behavior with respect to the change of $\sigma_{1}$, the level of the multiplicative chaos (noise), necessarily occurs only in the presence of the additive noise due to the nature of this competition. These features are generic and universal in a general class of systems displaying on-off intermittency with symmetry breaking. Multiplicative stochastic res- 
onance has been studied by Gammaitoni et al [15] in a multiplicatively driven bistable system with $\lambda=1$. In that case, the system is out of the regime of on-off intermittency, and consequently cannot display the property of (super)sensitivity, and the resonance with respect to the change of $\lambda$ was not resported.

\section{INFORMATION GAIN}

Now consider the system from the viewpoint of transmission and amplification of a weak signal $p s(t)$ contaminated with channel noise $\sigma_{2} e(t)$ through a system displaying on-off intermittency with symmetry-breaking. It is very interesting and practically important that more information about the signal may be obtained from the output $X(t)$ than from the noisy input $p s(t)+\sigma_{2} e(t)$ itself, besides the fact that the weak signal has been amplifyed to a level discernible with a low resolution detector. To examine the information gain, we compare $C$ with the correlation between the signal $p s(t)$ and the total noisy input $p s(t)+\sigma_{2} e(t)$, i.e. $C_{i n}=R / \sqrt{1+R^{2}}$. $C, C_{i n}$ and their difference are shown in Fig. 4. $C$ comes to a saturated value for $R \geq 1$, where the noise-induced transitions between $x>0$ and $x<0$ in short time-scales are rarely manifested by large bursts. This value does not approach 1.0 as $C_{i n}$ due to an average time-delay between $X(t)$ and $s(t)$ induced by the relaxation time which is longer for weaker signal. Clearly, appreciable information gain is obtained by the system in a broad range of $R$, and an optimal gain is found at a certain $R$ value. If taking into account the effect of the time-delay between $X(t)$ and $s(t)$, e.g. by defining $C_{\tau}$ as the maximum of the correlation between $X(t)$ and $s(t-\tau)$, the information gain region can be wider (Fig. 4).

To conclude, we demonstrate interesting universal features of robustness of supersensitivity, resonance and information gain in a class of nonlinear system subjected to a weak modulation. These systems present a new mechanism of resonant behavior compared to conventional stochastic resonance. While intermittent loss of synchronization may be harmful for any applications employing high-quality synchronization [5], the features found in this letter are meaningful for potential applications of on-off intermittency. On-off intermittency has been demonstrated in many experimental systems and we believe that the behaviors reported in this work can be tested in physical experiments.

This work is supported in part by grants from the Hong Kong Research Grants Council (RGC) and the Hong Kong Baptist University Faculty Research Grant (FRG).
[1] Ying-Cheng Lai, Phys. Rev. E 53, R4267, (1996).

[2] E. Ott and J. C. Sommerer, Phys. Lett. A 188, 39 (1994).

[3] T. Yamada and H. Fujisaka, Prog. Theor. Phys. 70, 1240 (1983); A. S. Pikovsky, Z. Phys. B. 55, 149 (1984); L. M. Pecora and T. L. Carroll, Phys. Rev. Lett. 64, 821 (1990); K. Josic, Phys. Rev. Lett. 80, 3053 (1998)

[4] P. Ashwin, J. Buescu, and I. Stewart, Phys. Lett. A 193, 126 (1994). S. C. Venkataramani, B. R. Hunt, E. Ott, D. J. Gauthier, and J. C. Bienfang, Phys. Rev. Lett. 77, 5361(1996).

[5] J. F. Heagy, T. L. Carroll, and L. M. Pecora, Phys. Rev. E 52, R1253 (1995); D. J. Gauthier and J. C. Bienfang, Phys. Rev. Lett. 77, 1751 (1996).

[6] L. Yu, E. Ott, and Q. Chen, Phys. Rev. Lett. 65 , 2935 (1990); N. Platt, E. A. Spiegel, and C. Tresser, Phys. Rev. Lett. 70, 279 (1993); N. Platt, S. M. Hammel, and J. F. Heagy, Phys. Rev. Lett. 72, 3498 (1994); J. F. Heagy, N. Platt, and S. M. Hammel, Phys. Rev. E 49, 1140 (1994); Y. H. Yu, K. Kwak, and T. K. Lim, Phys. Lett. A 198, 34 (1995); A. Cenys, A. Namajunas, A. Tamasevicius, and T. Schneider, Phys. Lett. A 213, 259 (1996); H. L. Yang and E. J. Ding, Phys. Rev. E 54, 1361 (1996).

[7] A. S. Pikovsky and Grassberger, J. Phys. A: Math. Gen. 24, 4587 (1991).

[8] For example, K. M. Cuomo and A. V. Oppenheim, Phys. Rev. Lett. 71 , 65 (1993).

[9] N. Platt, S. M. Hammel, and J. F. Heagy, Phys. Rev. Lett. 72, 3498 (1994).

[10] A. Cenys, and H. Lustfeld, J. Phys. A 29, 11 (1996).

[11] E. Ott, C. Grebogi and J. A. Yorke, Phys. Rev. Lett. 64, 1196 (1990). S. Hayes, C. Grebogi and E. Ott, Phys. Rev. Lett. 70 , 3031 (1993).

[12] S. L. Ginzburg and M. A. Pustovoit, Phys. Rev. Lett. 80, 4840 (1998).

[13] T. Zhou, F. Moss and A. Bulsara, Phys. Rev. A 45, 5394 (1992).

[14] L. Gammaitoni, P. Hänggi, P. Jung, and F. Marchesoni, Rev. Mod. Phys. 70, 223 (1998).

[15] L. Gammaitoni, F. Marchesoni, E. Menichella-Saetta, and S. Santucci, Phys. Rev. E 49, 4878 (1994).

FIG. 1. (a) A typical time series of $x(t)$ in the noisy environment with $\sigma_{2}=10^{-6}$ : upper panel, $R=0$, and lower panel, $R=0.5$. The dotted line is $s(t) . \lambda=-0.02, T=2000$, (b) Numerical estimated $\langle x\rangle$ as a function of $R$ (stars) compared to the analytical estimation of Eq. (1) (line). $\lambda=0$, $b=1$ and $p=10^{-7}$.

FIG. 2. Resonant behavior with respective to $\lambda=a-1$; $b=1$.

FIG. 3. Resonant behavior with respective to $b$. A resonance occurs when $\sigma_{2} \neq 0$.

FIG. 4. An illustration of information gain. $\lambda=0, b=1$. 
Fig. 1
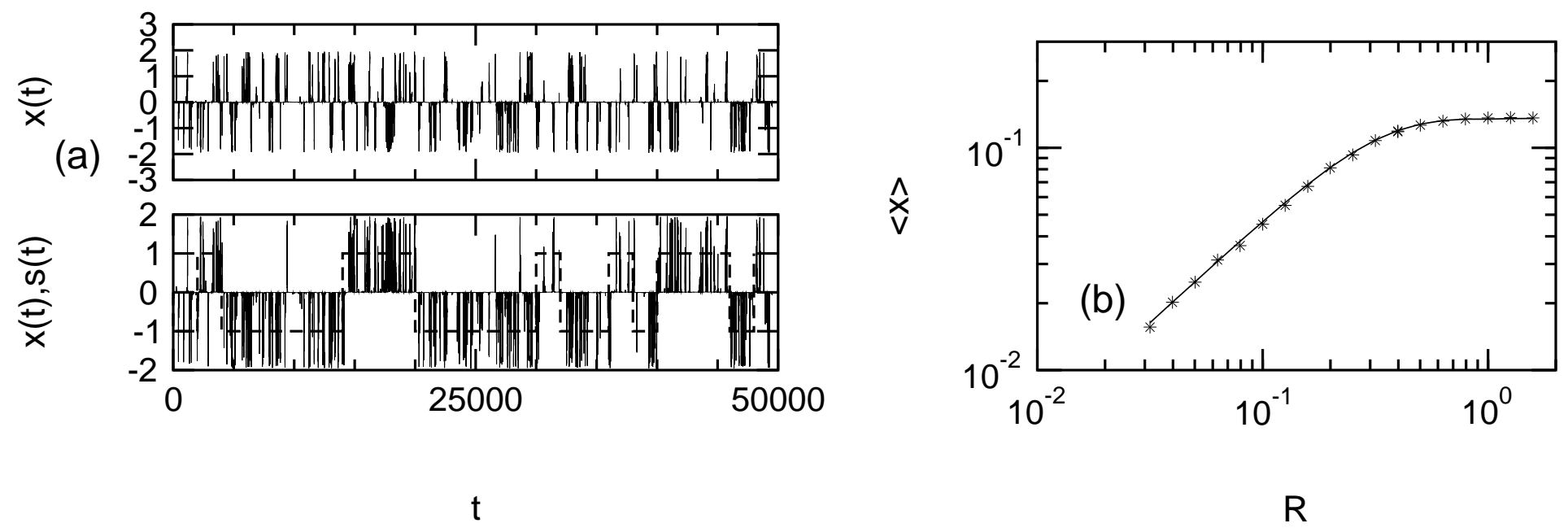
Fig. 4

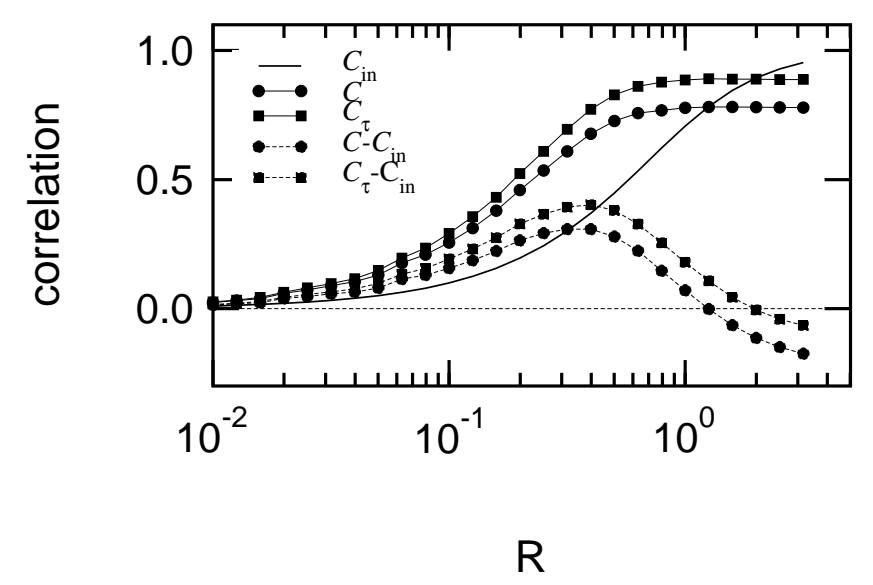


Fig. 2

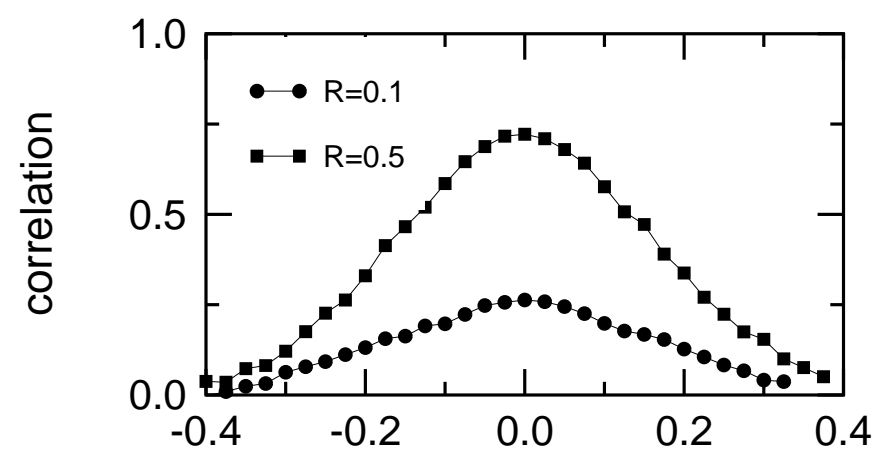

$\lambda$
Fig. 3

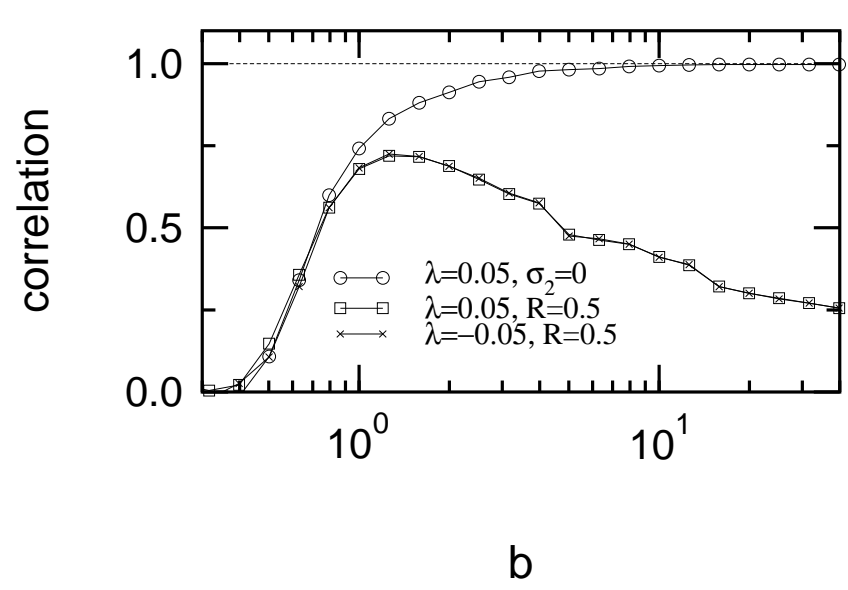

